\title{
Skin lesions of 52-year-old male butcher with delayed diagnosis and treatment: What is your diagnosis?
}

\author{
Gülcan Saylam Kurtipek ${ }^{1}$, Ercan Kurtipek ${ }^{2}$ \\ ${ }^{1}$ Department Of Dermatology, Konya Training and Research Hospital, Konya, Turkey \\ ${ }^{2}$ Department Of Chest Disease, Konya Training and Research Hospital, Konya, Turkey
}

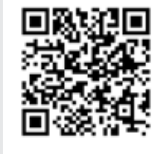

Received Date: 06.05 .2014 Accepted Date: 07.05.2014

\section{Address for correspondence}

Gülcan Saylam Kurtipek, Konya Eğitim ve Araştırma Hastanesi, Dermatoloji Bölümü, Konya, Türkiye

E-mail: gsaylamkurtipek@yahoo.com

(C) Copyright 2014 Turkish Respiratory Society (TRS) Eurasian J Pulmonol 2014

DOI: $10.5152 /$ ejp.2014.91300
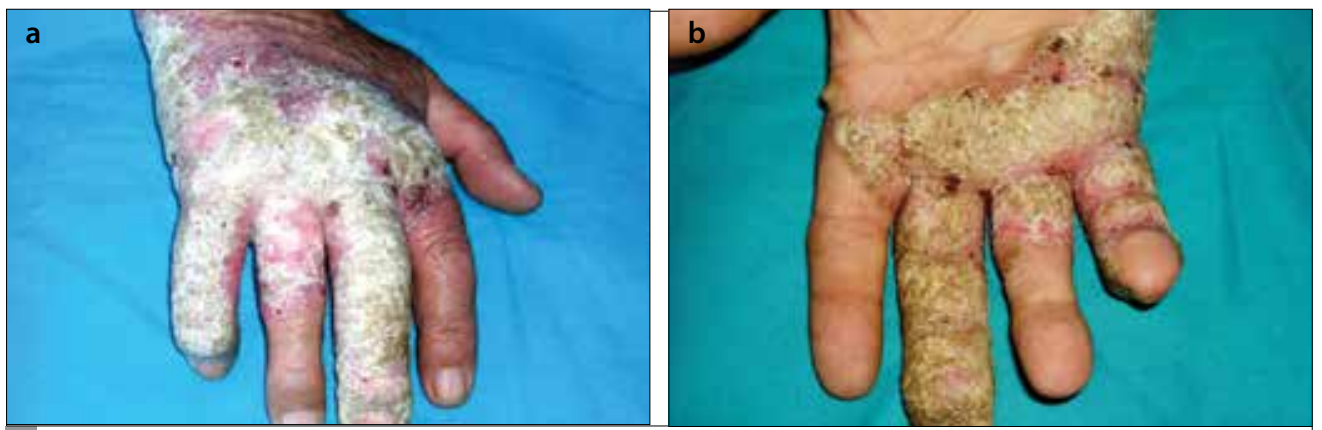

Figure 1.a, b. On the right dorsal surface of the hand, a squamous hyperkeratotic, well-delimited, approximately $15 \times 20-\mathrm{cm}$ plaque lesion with verrucous appearance and flesh-coloured (a). On the volar surface of right hand, a plaque lesion with verrucous appearance.

A 52-year-old male patient, who had been a butcher for a long time, was admitted to the Dermatological and Venereal Diseases Outpatient Clinic due to a lesion that had first occurred on the fingers of his right hand about $25-30$ years ago and had gradually extended to its current shape over time. In the investigation of the patient's medical history, it was found that he had not benefited from the previous therapies with topical agents. Dermatological examination revealed a squamous hyperkeratotic, well-delimited, approximately $15 \times 20-\mathrm{cm}$ plaque lesion with verrucous appearance and flesh-coloured on the volar and dorsal surfaces of the right hand (Figure 1a, b).

Skin biopsy was performed for the diagnosis of the patient whose routine blood test and chest radiography results were found to be within normal intervals. During histopathological examination of the lesion, hyperkeratosis, acanthosis, and papillomatosis in the epidermis, mononuclear inflammatory cell

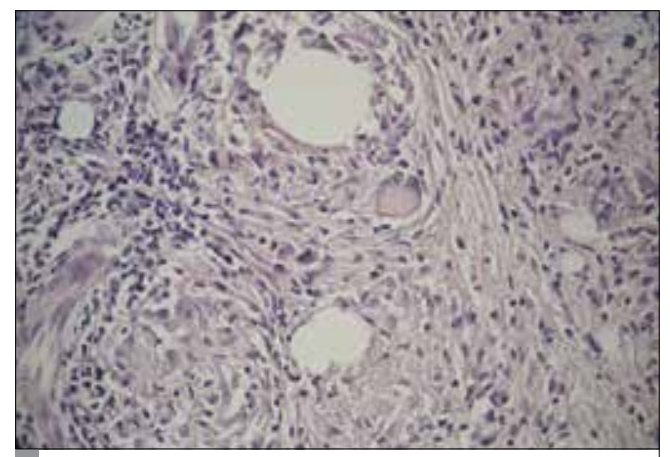

Figure 2. In histopathological examination of the lesion, hyperkeratosis, acanthosis, and papillomatosis in the epidermis, mononuclear inflammatory cell infiltrate just below the epithelium, and granulomatous inflammation and Langhans-type giant cells with necrosis in the middle dermis were observed. infiltrate just below the epithelium, and granulomatous inflammation and giant cells with necrosis in the middle dermis were observed (Figure 2).

What is the possible diagnosis after the evaluation of the patient's medical history and the biopsy results?
a) Skin involvement in sarcoidosis
b) Tuberculosis verrucosa cutis
c) Lymphoma
d) Deep fungal infection
e) Hypertrophic lichen planus 

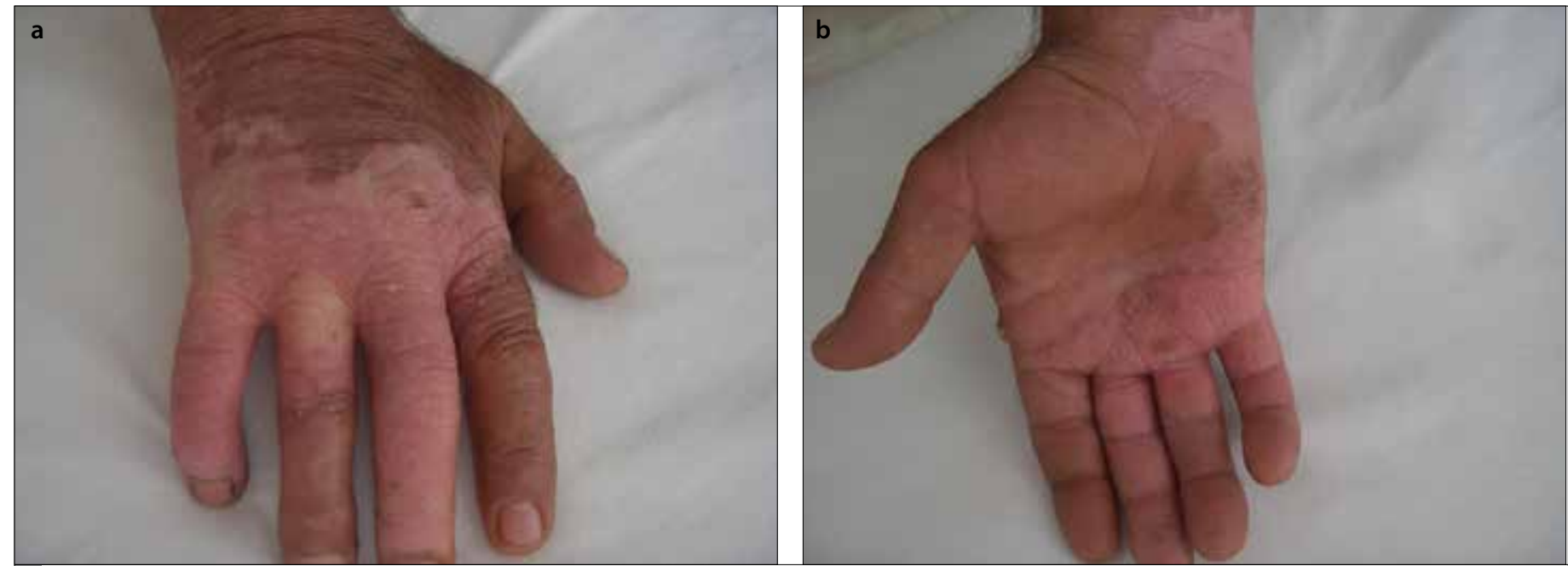

Figure 3. a, b. Post-treatment complete regression of the lesion in the hand dorsal leaving post-inflammatory hypopigmentation (a). Post-treatment post-inflammatory hyperpigmentation on the volar surface of the right hand (b).

\section{Correct answer b: Tuberculosis verrucosa cutis}

Tuberculosis verrucosa cutis (TBVC) is a paucibacillary form of cutaneous tuberculosis caused by exogenous re-infection in previously sensitised individuals. It is also known as warty tuberculosis, anatomist's wart, butcher's wart, verruca necrogenica, and lupus verrucosus. It is contaminated by the inoculation of bacilli into the skin in the individuals with moderate or high immunity. Inoculation occurs at the sites of minor wounds or abrasions, and rarely in the patient's sputum. Most of the time, the responsible agent is mycobacterium tuberculosis hominis and less frequently, mycobacterium bovis or mycobacterium avium. TBVC is a rare form of cutaneous tuberculosis in western countries, which can be quite common in the continent of Asia. In Europe, the affected parts of the body are mostly the hands, but in Eastern countries, the knees, ankles and buttocks are more often affected, which are generally areas that are exposed to trauma $(1,2)$. From a clinical aspect, it first presents as a small indurated nodule and then gradually extends, producing an irregular reddish-brown warty plaque. In some areas, healing is observed with an atrophic pale scar. Its differential diagnosis must clinically be made by deep fungus infections such as blastomycosis and chromomycosis, lichen simplex chronicus, and hypertrophic lichen planus $(3,4)$.

Its histopathological features are characterised by acanthotic papillomatosis with marked hyperkeratosis and granulomatous infiltrate caseous necrosis, consisting of neutrophils and lymphocytes in the deeper dermis $(5,6)$. In the biopsy sample taken from our case, hyperkeratosis, acanthosis, and papillomatosis in the epidermis, mononuclear inflammatory cell infiltrate just below the epithelium, and granulomatous inflammation and Langhans-type giant cells with caseous necrosis in the middle dermis were observed.

In the literature, there are many case studies demonstrating that polymerase chain reaction (PCR) is useless for determining mycobacterium tuberculosis from cutaneous tuberculosis. PCR is often negative in the paucibacillary form of cutaneous tuberculosis. For cases with a strong suspicion of tuberculosis, despite negative laboratory test results, anti-tuberculous therapy can be initiated. After this standard anti-tuberculous treatment, healing is expected to occur within $4-5$ months $(7,8)$.

In our case, after the clinical and histopathological diagnosis of a patient with TBVC, quadruple anti-tuberculous therapy (isoniazide 300 $\mathrm{mg} /$ day, rifampicin $600 \mathrm{mg} /$ day, pyrazinamide $1500 \mathrm{mg} /$ day, ethambutol $1500 \mathrm{mg} /$ day) was applied for the first two months and, subsequently, the patient was exposed to binary maintenance therapy for the following four months (isoniazide $300 \mathrm{mg} /$ day, rifampicin $600 \mathrm{mg} /$ day). At the end of treatment, the lesion in the right hand regressed completely leaving post-inflammatory hypopigmentation (Figure 3).

The progression of TBVC is slow. If can become chronic if it is not treated. There are only a few cases where lesions are present for more than 20 years. In our case, the patient had experienced the lesions for about 25-30 years, and responded to the standard anti-tuberculous treatment within a short period of 2 months (Figure $3 a, b)$.

\section{Ethics Committee Approval: N/A.}

Informed Consent: Written informed consent was obtained frompatient who participated in this study.

Peer-review: Externally peer-reviewed.

Author Contributions: All authors contributed equally during the preparation of this manuscript.

Conflict of Interest: No conflict of interest was declared by the authors.

Financial Disclosure: The authors declared that this study has received no financial support.

\section{REFERENCES}

1. Tappeiner $G$, Wolff $K$. Tuberculosis and other mycobacterial infections. In: Freedberg IM, Eisen AZ, Wolff K, et al. Eds. Fitzpatrick's Dermatology in General Medicine, 6th ed. New York: Mc Graw-Hill, 2003: 1936-7.

2. Wong KO, Lee KP, Chui SF. Tuberculosis of the skin in Hong Kong (a review of 160 cases). Br J Dermatol 1986: 424-9.

3. Foo $\mathrm{CCl}$, Tan $\mathrm{HH}$. A case of tuberculosis verrucosa cutis-undiagnosed for 44 years and resulting in fixed-flexion deformity of the arm. Clinical Exp Dermatology 2005; 30: 149-51.

4. Gruber PC, Whittam LR, DuVivier A. Tuberculosis verrucosa cutis which remained on the sole and foot. Clin Exp Dermatol 2002; 27: 188-91.

5. Pereira MB, Gomes MK, Pereira F. Tuberculosis verrucosa cutis associated with tuberculous lymphadenitis. Int J Dermatol 2000; 39: 856-8.

6. Masellis P, Gasparini G, Caputo R, Alessi E. Tuberculosis verrucosa cutis which remained undiagnosed for fory-three years. Dermatology 1995;192: 145-8.

7. Tan Sh, Tan BH, Goh CL, Tan KC, Tan MF, Nq WC, et al. Detection of Mycobacterium tuberculosis DNA us inpolymerasechainreaction in cutaneous tuberculosis and tuberculids. Int J Dermatol 1999; 38: 122-7.

8. Utaş, Güler E, Postgil Yılmaz S. A Case of tuberculosis verrucosa cutis, misdiagnosedand treated as a wart. Türkiye Klinikleri J Med Sci 2012; 32: 822-6. 